\title{
Role of sonosalpingogram in correlation to hysterosalpingogram in assessment of infertility
}

\author{
Rajneesh Madhok*, Vichi Taneja
}

\begin{abstract}
Department of Radiodiagnosis, Shri Ram Murti Smarak Institute of Medical Sciences, Bareilly, Uttar Pradesh, UP,
\end{abstract} India

Received: 07 April 2016

Accepted: 07 May 2016

\section{*Correspondence:}

Dr. Rajneesh Madhok,

E-mail:drmadhok@gmail.com

Copyright: () the author(s), publisher and licensee Medip Academy. This is an open-access article distributed under the terms of the Creative Commons Attribution Non-Commercial License, which permits unrestricted non-commercial use, distribution, and reproduction in any medium, provided the original work is properly cited.

\section{ABSTRACT}

Background: This study was performed to evaluate the patency of fallopian tubes with hysterosalpingographyand sonosalpingographyin females with complaint of infertility and to correlate the results of sonosalpingogram with that of hysterosalpingogram in assessment of tubal patency in infertility.

Methods: Females with complaint of infertility were evaluated by HSG (Hysterosalpingography) and SSG (Sonosalpingography) between 7th and 12th day of menstrual cycle. HSG was carried out using Siemens fluoroscopy machine. The flow of contrast was followed and tubal patency was assessed. SSG was carried out using Siemens ACUSON X300, with transvaginal probe (4-9MHz). Normal saline was infused slowly and the endometrial cavity was evaluated. Then color flow was used to evaluate each of the adnexa for free spillage of saline (waterfall sign) and results of both the techniques were correlated.

Results: Altogether 94 tubes were evaluated by HSG \& SSG each. On HSG, out of 94, 65 tubes were patent and 29 tubes showed blockage. On SSG, 68 tubes were patent and 26 tubes showed blockage. Results of both the techniques were correlated by Kappa value which came out to be 0.923 . There was very good agreement between SSG and HSG. Conclusions: There was 92.3\% agreement (KAPPA value $=0.923$, Standard error $=0.437,0.95 \mathrm{CI}=0.8373-1$ ) between SSG and HSG which suggests that SSG is at least similar to HSG in its effectiveness for evaluating tubal patency and has the potential to replace HSG as routine, first-line outpatient infertility investigation.

Keywords: Infertility, Hysterosalpingogram, Sonosalpingogram, Fallopian tubes

\section{INTRODUCTION}

Infertility is "a disease of the reproductive system defined by the failure to achieve clinical pregnancy after 12 months or more of regular unprotected sexual intercourse. ${ }^{1}$ Infertility can be primary or secondary.

When a woman is unable to ever bear a child, either due to the inability to become pregnant or the inability to carry a pregnancy to a live birth she would be classified as having primary infertility. Thus women whose pregnancy spontaneously miscarries, or whose pregnancy results in a stillborn child, without ever having had a live birth would present with primarily infertility.
When a woman is unable to bear a child, either due to the inability to become pregnant or the inability to carry a pregnancy to a live birth following either a previous pregnancy or a previous ability to carry a pregnancy to a live birth, she would be classified as having secondary infertility. Thus those who repeatedly spontaneously miscarry or whose pregnancy results in a stillbirth, following a previous pregnancy or a previous ability to do so, are then not unable to carry a pregnancy to a live birth would present with secondary infertility. ${ }^{2}$

The causes of female infertility can be broadly categorized into the following: 
Uterine causes - Congenital anomalies, infections, uterine synechiae, focal lesions, intrauterine scar, cervical stenosis, reduced uterine perfusion and alterations in endometrial thickness and vascularity.

Ovarian causes - Follicular and ovulation abnormalities, stromal vascularity, and endometriosis. Tubal causes Infections, obstruction. $^{3}$

The incidence of tubal disease in infertility varies from country to country. In India it has been estimated to be about $40 \%$. The prevalence of pelvic inflammatory disease, genital tract tuberculosis, and chronic infection is quite common in our country and hence the incidence of tubal factor in infertile women is high. ${ }^{4}$

Precise evaluation of the patency of fallopian tubes is an important step in infertility assessment.There are multiple etiologic factors responsible for the involvement of the fallopian tube in infertility, which include tubal damage from pelvic inflammatory disease (PID), endometriosis, use of intrauterine devices, a history of a perforated appendicitis, ectopic pregnancy, and septic abortion. Tubal adhesions and tubal obstruction can result in infertility. ${ }^{5}$ Gonorrhoea, chlamydial infection or salpingitis following septic abortion or puerperal infections are amongst the common causes of blockage of fallopian tubes. ${ }^{6}$

Proximal tubal occlusion can be associated with mild peritoneal endometriosis. Distal tubal blockage, which is commonly caused by pelvic inflammatory disease, is usually associated with distension of the ampullary portion of the fallopian tube (hydrosalpinges) and variable degree of loss of the internal mucosal folds. ${ }^{7}$

In the past 20 years, Fallopian tube patency has been studied by different modalities such as X-Rayhysterosalpingography (X-Ray HSG), ultrasonography hysterography or Sonosalpingography (SSG), laparoscopic hysteroscopy, hysteroscopic guided falloscopy, among them, X-Ray HSG named as hysterosalpingography (HSG) remain frequently used procedure. $^{8}$

Hysterosalpingography (HSG) is the radiographic evaluation of the uterus and fallopian tubes and is used predominantly in the evaluation of infertility. The primary role of HSG is in the evaluation of the fallopian tubes. ${ }^{9}$ HSG was first performed in 1910, by Rindfleish, by injecting a bismuth solution in the uterine cavity. ${ }^{10}$ In 1914, the first report on HSG for determining tubal patency with an oil-soluble contrast medium as an outpatient procedure was published by Carey. ${ }^{11}$ Since then HSG is widely used for tubal evaluation in infertile women. This method is fairly accurate in detecting tubal disease, is safe, not much expensive and may potentially be associated with increased pregnancy rates. ${ }^{12}$
Sonosalpingography (SSG), an ultrasound-based technique for the visualization of the fallopian tubes using normal saline as agent, has been proposed as an alternative to hysterosalpingography (HSG) to assess tubal patency in the initial workup of infertile couples. ${ }^{13}$ This test is used as a basic screening test for evaluating tubal patency in all infertile cases and helps in screening cases for laparoscopy.Richman et al were first to report on the abdominal sonographic evaluation of uterus \& tubal patency after injecting isotonic solution through special intra-uterine catheter (Harris uterine injector). ${ }^{8}$

SSG and HSG are both short, well-tolerated outpatient procedures. ${ }^{14}$ The mean dose-area product (DAP) for a complete HSG examination is $2.05 \mathrm{~Gy} \mathrm{~cm}^{2}$. ${ }^{15}$ Entrance surface doses and ovarian doses range from $2.3 \mathrm{mGy}$ and $0.5 \mathrm{mGy}$ in digital systems to $13.1 \mathrm{mGy}$ and $3.1 \mathrm{mGy}$ in analog systems, respectively. ${ }^{16}$ In contrast, Sonohysterosalpingography eliminated the need for iodinated contrast and ionizing radiations with their associated risks.

Laparoscopy and dye insufflation test is generally regarded as being the gold standard for checking the patency of the fallopian tubes $\&$ for assessment of pelvic health but is an invasive diagnostic method that requires general anesthesia and carries the risk of severe adverse effects, including injury of pelvic blood vessels, intestinal loops, and the urinary bladder. It also does not provide an assessment of the uterine cavity.

This implies the need for a diagnostic tool with high sensitivity and specificity to distinguish an actual fallopian tube obstruction as an alternative to HSG \& to decrease the need for laparoscopy \& dye insufflations test. $^{17}$

\section{METHODS}

Females with the complaint of primary/ secondary infertility were evaluated by HSG \& SSG between $7^{\text {th }}$ and $12^{\text {th }}$ day of their menstrual cycles.

Inclusion criteria includedinfertile females, Age 20-40yrs with normal menstrual cycles

Exclusion criteria includedactive PID(Pelvic inflammatory disease), active genital tuberculosis, medical or hormonal dysfunction, oligospermia/ azoospermia in male partner

The patient was instructed to abstain from sexual intercourse from the time menstrual bleeding ends until the day of the study to avoid a potential pregnancy. The procedure was explained to the patients and informed consent obtained. Because patients may experience cramping during the examination, they were given $2 \mathrm{ml}$ IM buscopan 30 minutes prior to the procedure. 
HSG was carried out using siemens fluoroscopy machine, Luminos Select, and iodine contrast media .The patient was placed in the lithotomy position at the edge of the fluoroscopy table. A speculum was inserted into the vagina. The cervix was localized and cleansed with povidone- iodine solution. The anterior lip of the cervix was held with vulsellum and gentle traction was applied. Leech Wilkinson cannula was inserted into the cervical canal. 5-10ml of contrast medium was slowly injected through the cervical canal into the uterine cavity. By fluoroscopy the flow of contrast was followed and the uterine cavity and the fallopian tubes were visualized and tubal patency was assessed in each patient.

SSG was carried out using siemens Acuson X300, with transvaginal probe $(4-9 \mathrm{MHz})$. The patient was placed in the lithotomy position at the edge of the ultrasound table. A Cusco speculum was gently inserted in vagina and the cervix washed with an antiseptic solution. The anterior lip of the cervix was grasped with a vulsellum and the 8 French pediatric foley's catheter with guide wire was inserted about $5 \mathrm{~cm}$ into the endocervix and passed above the level of the internal os. The balloon was then inflated with $3 \mathrm{ml}$ of isotonic saline solution. The catheter guide in foley's and the speculum were removed. The transvaginal probe was inserted and through the catheter 20-40 $\mathrm{ml}$ normal saline was infused slowly to distend the endometrial cavity. During the distension, and by the end of it, the cavity was evaluated. Then color flow was used to evaluate each of the adenexa for free spillage of saline (waterfall sign).

After completing both the procedures, results of SSG were correlated to the results of HSG.

\section{RESULTS}

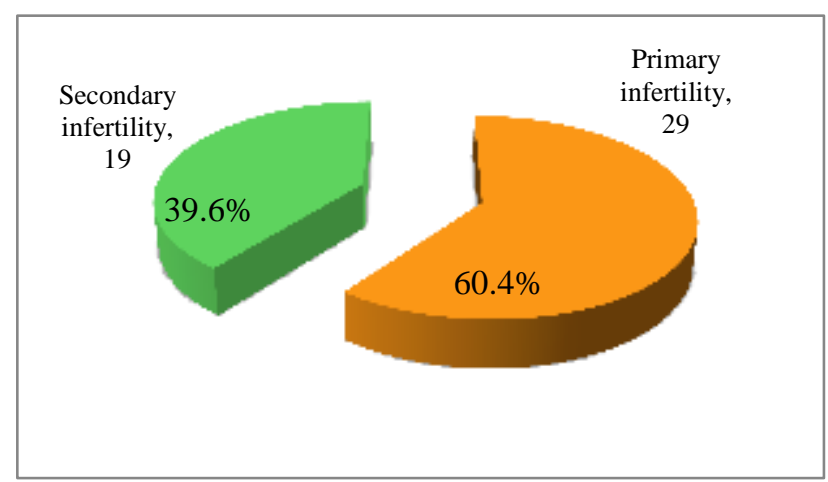

Figure 1: Primary versus secondary infertility.

50 patients of primary/secondary infertility were evaluated for patency of the fallopian tubes. Out of those, 2 patients were excluded because of intravasation of contrast on HSG. Total 48 patients were finally included in the study (Figure 1). Two of the patients demonstrated unicornuate uterus so, altogether 94 tubes were evaluated by HSG and SSG each.

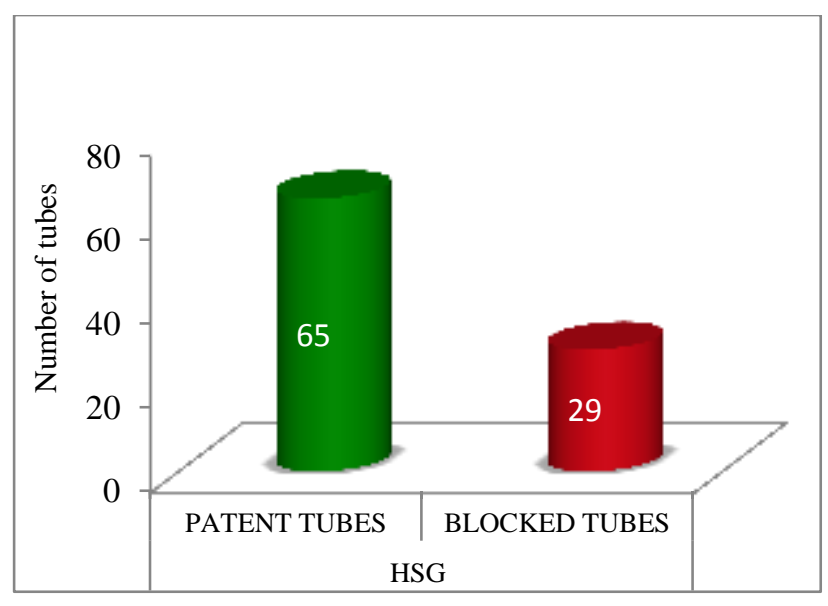

Figure 2: Imaging findings on HSG (Total number of tubes: 94).

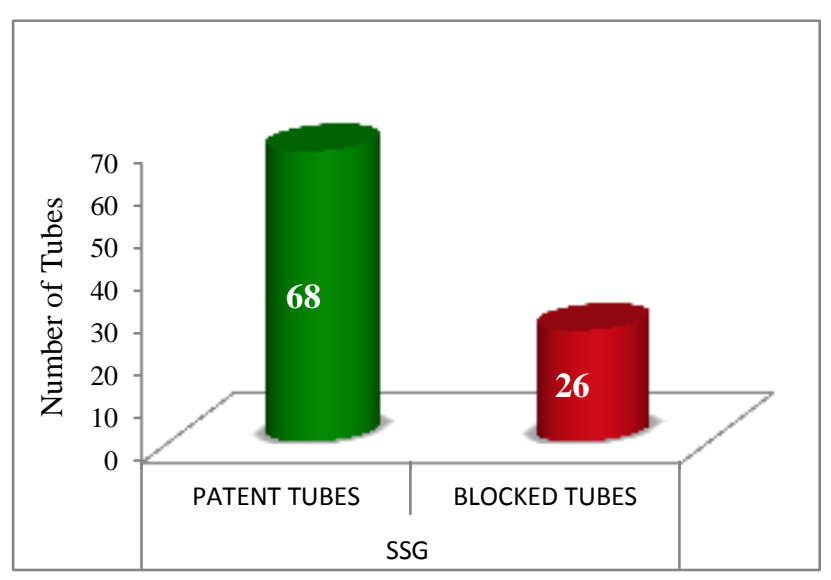

Figure 3: Imaging findings on SSG (Total number of tubes - 94).

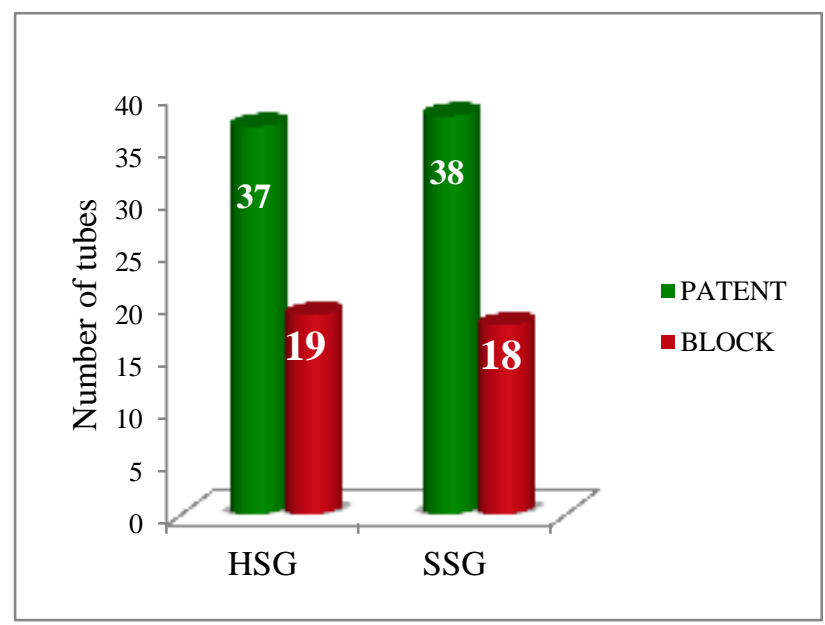

Figure 4: Tubal findings in primary infertility on HSG and SSG (56 tubes: 29 patients).

56 tubes of primary infertile patients were evaluated by HSG and SSG. One tube that showed blockage on HSG was interpreted as patent on SSG. 
38 tubes of secondary infertile patients were evaluated by HSG and SSG. 2 of the tubes that were interpreted as blocked on HSG were interpreted as patent on SSG.

Total 65 tubes showed patency and 29 tubes showed blockage on HSG (Figure 2), whereas on SSG, 68 tubes were patent and 26 tubes were blocked (Figure 3 ).

Therefore 65 tubes were interpreted as patent and 26 tubes were interpreted as blocked by HSG as well as SSG suggesting good agreement between the two techniques (Table 1).

Table 1: Assessment and correlation between SSG and HSG for tubal patency according to number of tubes.

\begin{tabular}{|lllll|}
\begin{tabular}{|l} 
Examination \\
technique
\end{tabular} & $\begin{array}{l}\text { Number } \\
\text { of tubes } \\
\text { patent }\end{array}$ & $\begin{array}{l}\text { Percentage } \\
(\%)\end{array}$ & $\begin{array}{l}\text { Number } \\
\text { of tubes } \\
\text { blocked }\end{array}$ & $\%$ \\
\hline HSG & 65 & 69.1 & 29 & 30.9 \\
\hline SSG & 68 & 72.3 & 26 & 27.7 \\
\hline
\end{tabular}

Table 2: Discrepancy between HSG and SSG.

\begin{tabular}{|lll|}
\hline Patients & HSG finding & SSG finding \\
\hline Patient A & Unilateral block & Bilateral patent \\
\hline Patient B & Bilateral block & Unilateral block \\
\hline Patient C & Bilateral block & Unilateral block \\
\hline
\end{tabular}

Table 3: Age profile of patients.

\begin{tabular}{|lllllll|}
\hline $\begin{array}{l}\text { Age } \\
\text { in } \\
\text { years }\end{array}$ & $\begin{array}{l}\text { No of } \\
\text { patients }\end{array}$ & \multicolumn{2}{l}{$\begin{array}{l}\text { Primary } \\
\text { infertility }\end{array}$} & \multicolumn{2}{l}{$\begin{array}{l}\text { Secondary } \\
\text { infertility }\end{array}$} & $\%$ \\
\hline $20-23$ & 6 & 4 & $8.3 \%$ & 2 & $4.2 \%$ & $12.5 \%$ \\
\hline $24-27$ & 24 & 18 & $37.5 \%$ & 6 & $12.5 \%$ & $50 \%$ \\
\hline $28-31$ & 10 & 3 & $6.2 \%$ & 7 & $14.6 \%$ & $20.8 \%$ \\
\hline $32-35$ & 6 & 2 & $4.2 \%$ & 4 & $8.3 \%$ & $12.5 \%$ \\
\hline$>35$ & 2 & 2 & $4.2 \%$ & 0 & $0 \%$ & $4.2 \%$ \\
\hline Total & 48 & 29 & $60.4 \%$ & 19 & $39.6 \%$ & $100 \%$ \\
\hline
\end{tabular}

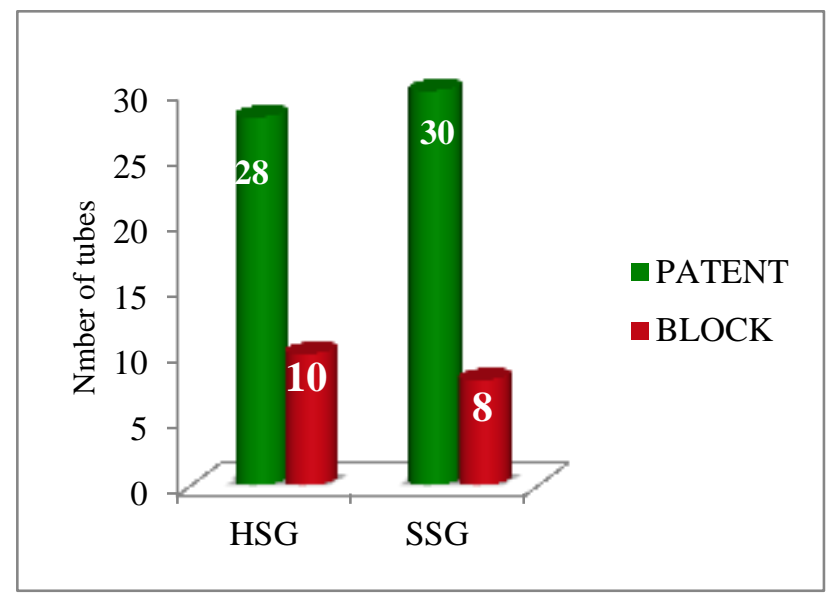

Figure 5: Tubal findings in secondary infertility on HSG and SSG (38 tubes: 19 patients).

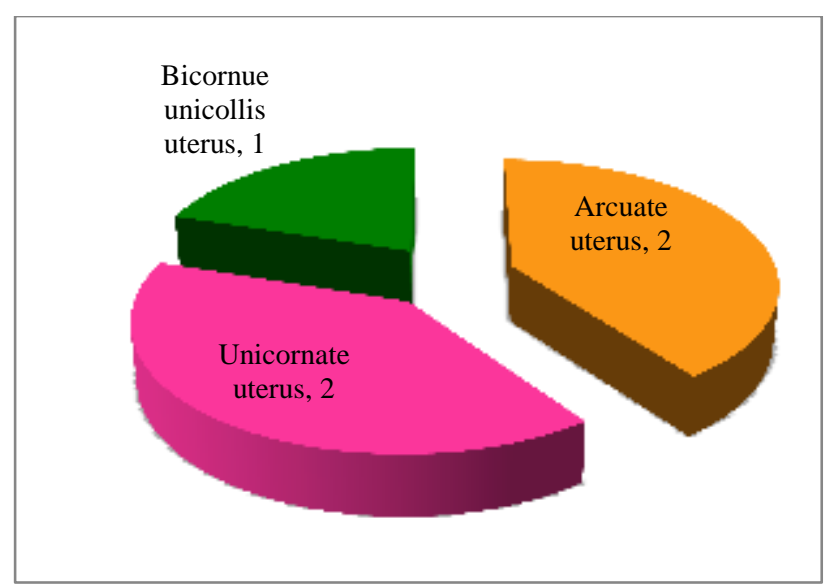

Figure 6: Associated imaging findings: HSG.

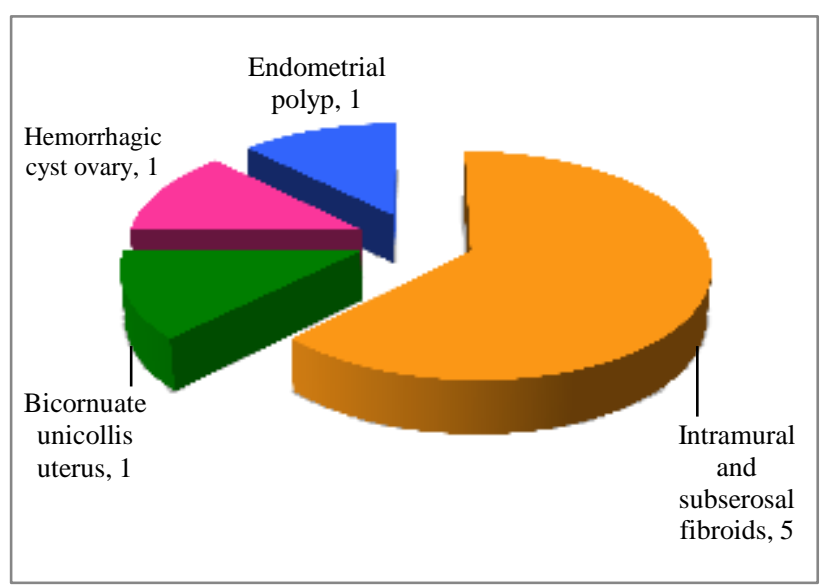

Figure 7: Associated imaging findings: SSG.

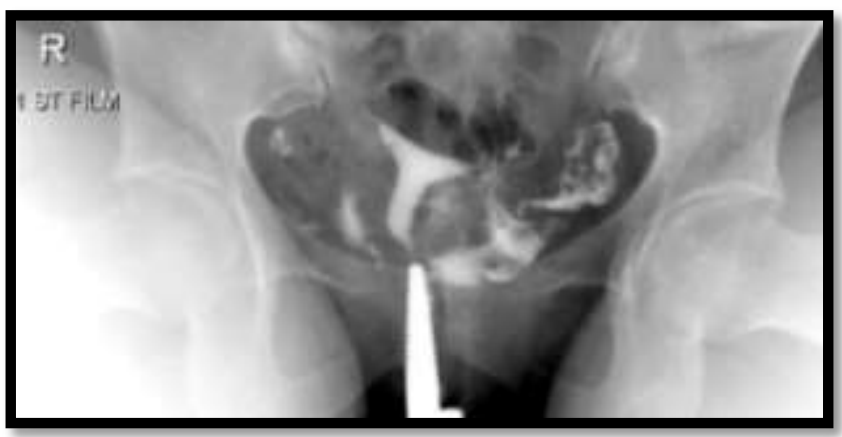

Figure 8: Normal hysterosalpingogram: spot radiograph obtained with uterus fully distended with contrast material, bilateral fallopian tubes are opacified with intraperitoneal spillage of contrast through the fimbrial ends.

Discrepancy of results was seen in 3 out of 94 tubes evaluated. These 3 tubes were interpreted as blocked on HSG, however on SSG, these demonstrated spillage of saline (waterfall sign) beautifully. Discrepancy of 2 tubes were seen in 2 patients who showed bilateral tubal block on HSG but unilateral tubal block on SSG and discrepancy of the $3^{\text {rd }}$ tube was seen in another patient who showed unilateral tubal block on HSG however SSG 
showed bilateral tubal patency in that patient. (Table 2). These patients were taken for laparoscopic chromopertubation and the findings of SSG were confirmed.

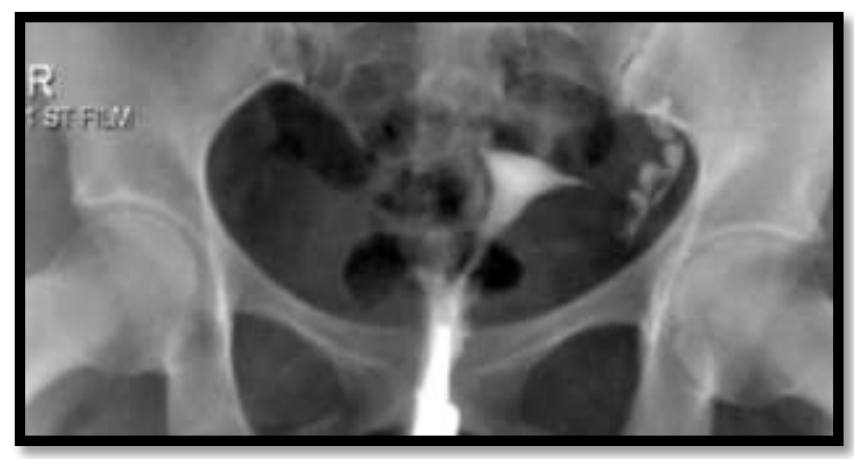

Figure 9: Hysterosalpingogram: spot radiograph showing non-opacification of right fallopian tube with absence of intraperitoneal contrast spillage suggesting right tubal block. However, opacification of

interstitial, isthmic and ampullary parts of left fallopian tube is seen with free spillage of contrast.

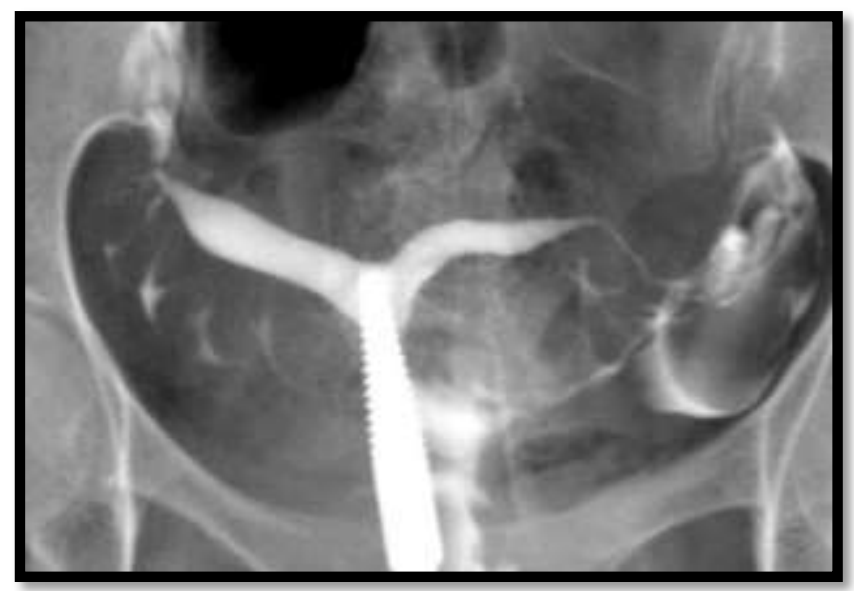

Figure 10: Hysterosalpingogram: spot radiograph showing bicornuate unicollis uterus with bilateral intraperitoneal free spillage of contrast.

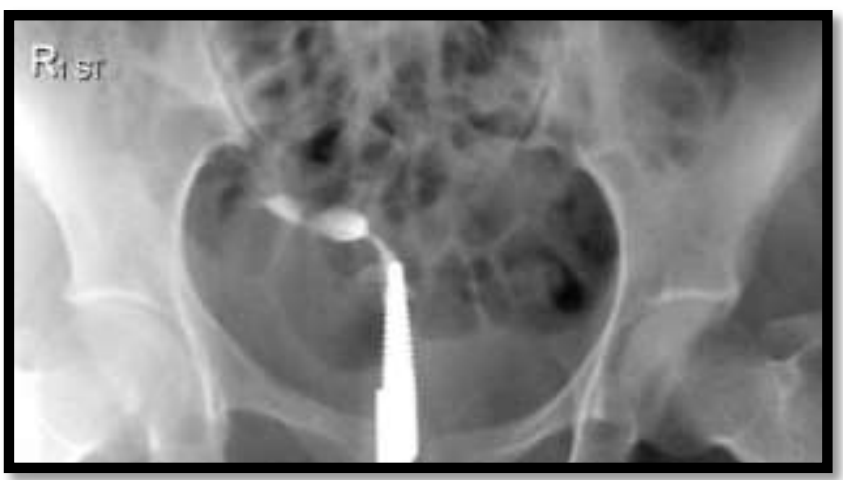

Figure 11: Hysterosalpingogram: spot radiograph showing unicornuate uterus seen with absence of opacification of fallopian tube and intraperitoneal spill.

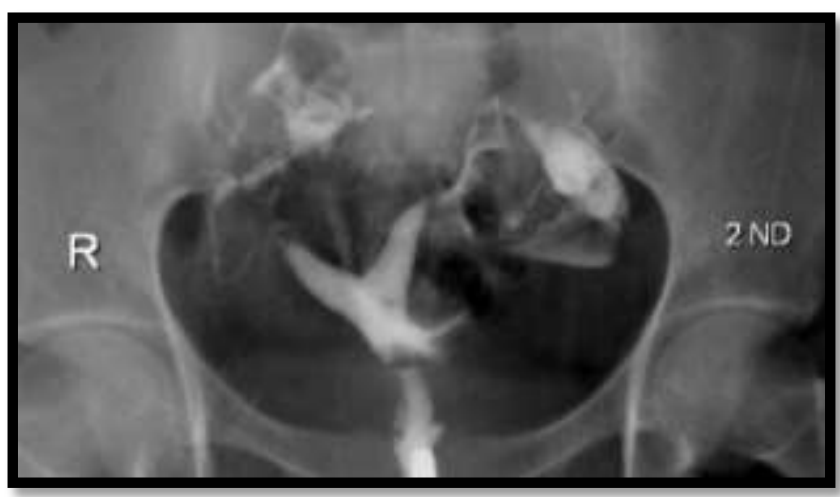

Figure 12: Hysterosalpingogram: spot radiograph showing arcuate uterus, distended endometrial cavity and opacified fallopian tubes with bilateral intraperitoneal spillage of contrast.

Altogether 94 tubes were evaluated by HSG \& SSG and the results of both the techniques were correlated by taking out Kappa value which was calculated to be 0.923 . There was very good agreement between SSG and HSG. Standard error was 0.437 and 0.95 CI was $0.8373-1$.

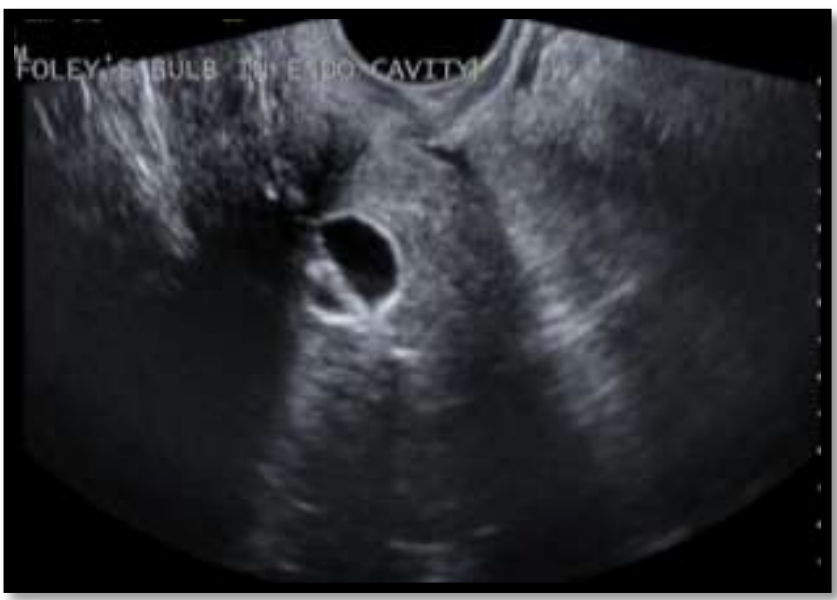

Figure 13: Transvaginal sonography showing foley's bulb in the endometrial cavity.

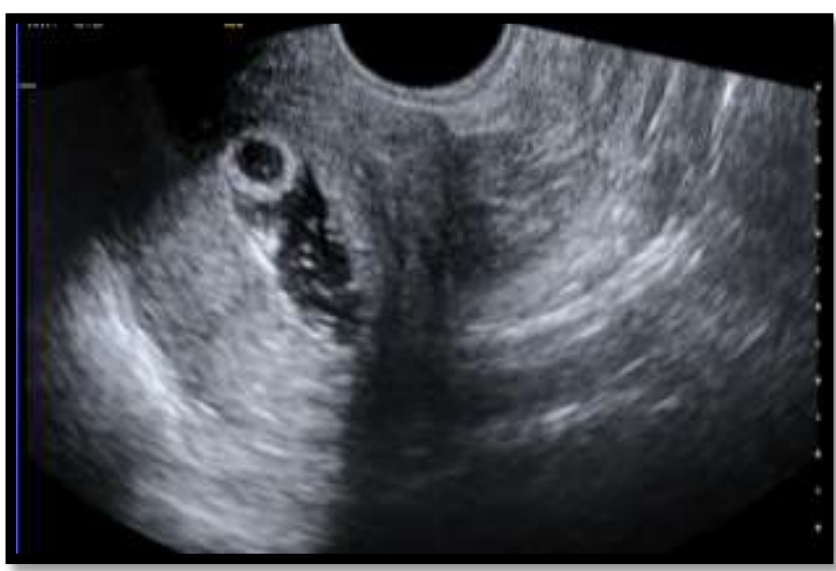

Figure 14: Sonosalpingogram: distended endometrial cavity with normal saline separating the walls of endometrium. 
Maximum numbers of primary infertile females were between 24-27 years and maximum numbers of secondary infertile females were between 28-31 years (Table 3).

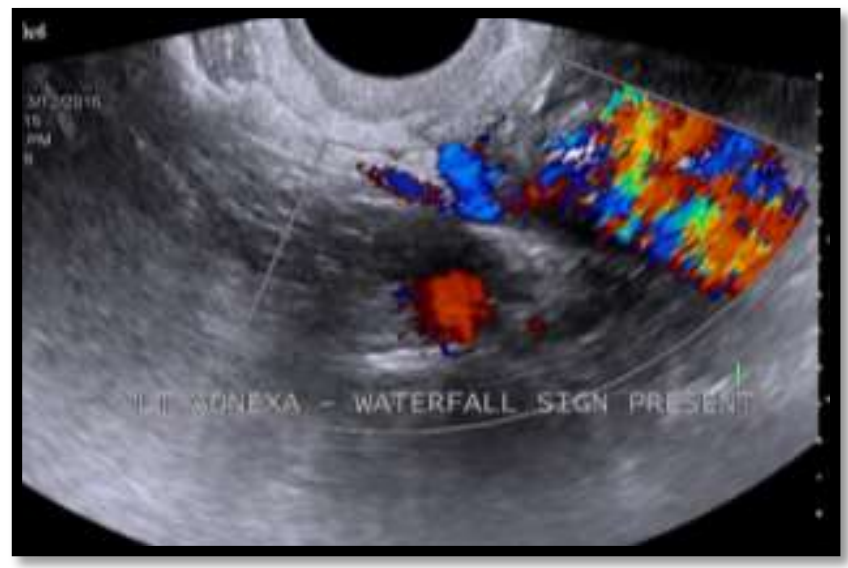

Figure 15: Sonosalpingogram: color bruit seen in left adnexa after the infusion of normal saline through foley's bulb seen as waterfall sign suggesting patency of the fallopian tube.

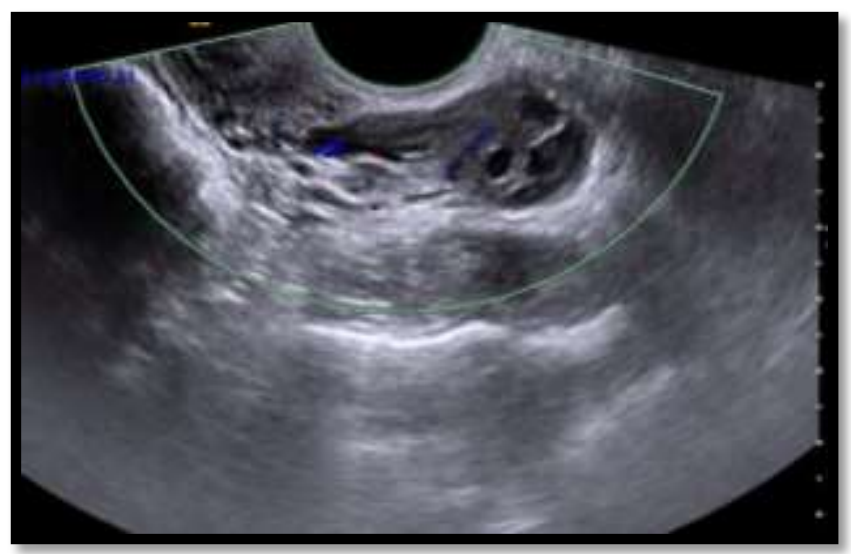

Figure 16: Sonosalpingogram showing absence of waterfall sign after infusion of normal saline through foley's bulb suggesting tubal block.

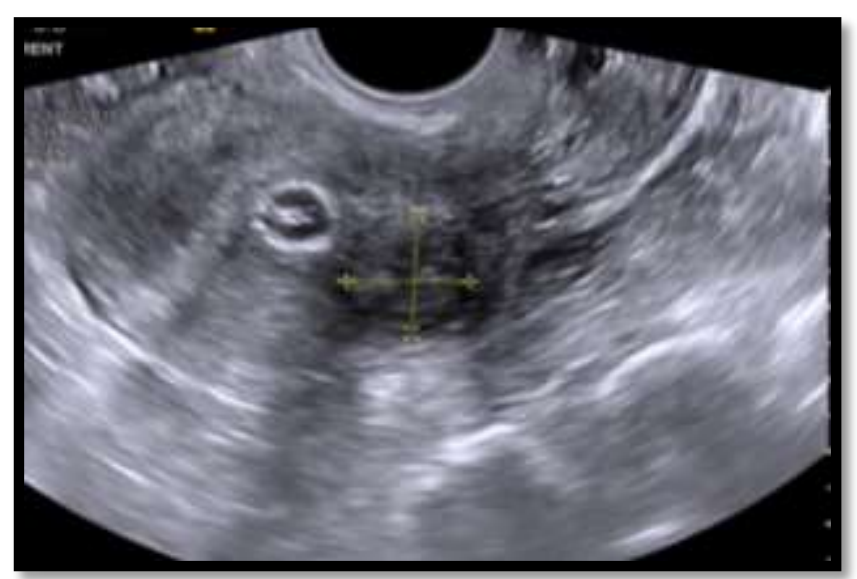

Figure 17: Intramural fibroid seen in the posterior wall of the uterus while performing SSG.

\section{DISCUSSION}

One of the major causes of infertility is blockage of the fallopian tubes. In the investigation and treatment of infertility, it is important to establish that the tubes are patent. This is necessary because any treatment, such as induction of ovulation and artificial insemination, given without making sure that the tubes are patent may be a futile effort. ${ }^{18}$ This study was conducted to bring into focus the value of sonoslpingogram in assessing tubal patency in order to overcome the radiation hazard associated with hysterosalpingogram (HSG), reduce cost of examination and encourage it as first-line out-patient procedure for management of female infertility by comparing the two studies.

There are certain merits and demerits of both the techniques. The congenital uterine anomalies like unicornuate, bicornuate and arcuate uterus are more clearly demonstrated by HSG, however, SSG provides more detailed view of the uterine cavity \& myometrium. HSG do not provide the view of myometrium, thus is less sensitive for fibroids and SSG do not allow the distinction between distal and proximal occlusion. SSG has the advantage over HSG of permitting concomitant visualization of the ovaries.

In our study $6.2 \%$ (3 out of 48 patients) of females showed tubal patency on SSG but tubal blockage on HSG. These patients underwent laparoscopic chromopertubation, which confirmed the findings of SSG. This may be because HSG is painful and a number of obstructions detected on HSG are not based on actual tubal conditions but are due to a transient spasm.

In our study, there was $92.3 \%$ agreement (Kappa value $=$ 0.923, Standard error $=0.437,0.95 \mathrm{CI}=0.8373-1$ ) between SSG and HSG which suggests that SSG is at least similar to or slightly better to HSG in its effectiveness for evaluating tubal patency.

Tüfekçi EC et al evaluated tubal patency by transvaginal sonosalpingography and on the basis of results concluded that transvaginal sonosalpingography, with its accuracy and safety, is a promising screening and diagnostic technique in the evaluation of tubal patency on ambulatory basis. ${ }^{19}$

Oguntoyinbo AE, Amok AOD and Komolafe reported that there is no statistically significant difference $(\mathrm{p}=$ 0.237 ) between the results of the two methods (HSG and SHSG). SHSG is found to be a reliable, relatively inexpensive diagnostic and therapeutic (guided hydrotubation) procedure in the management of female infertility. In our study we also found no significant difference between the two test but SSG showed slight better performance to demonstrate tubal patency over HSG 
Suttipichate $\mathbf{J}$ et al performed a prospective study to evaluate the characteristics of transvaginal saline sonosalpingography (SSG) for the assessment of tubal patency in comparison to the findings from the standard diagnostic laparoscopy with chromopertubation. The results confirm that transvaginal saline SSG is a simple, well-tolerated and reliable screening method for the assessment of tubal patency in an outpatient setting with minimal adverse effect. ${ }^{20}$ In our study, the results of 3 discrepancy tube blockage/ patency on HSG/SSG in with chromopertubation were in favor of transvaginal saline SSG result.

Case AM and Pierson RA studied Clinical use of sonohysterography in the evaluation of infertility and concluded that Sonohysterography can offer detailed assessment of the female pelvis, and has the potential to replace HSG as a routine, first-line infertility investigation. $^{21}$ Zvanca M, Andrei C examined patients of infertility by sono-hysterosalpingography\& checked their results by laparoscopy also concluded the same. ${ }^{22}$

Goynumer $\mathrm{G}$ et al evaluated and compared the diagnostic value of HSG with laparoscopy in assessment of fallopian tube patency in infertile women and concluded that HSG demonstrated reduced positive predictive value especially for bilateral proximal tubal occlusion. ${ }^{23}$ This was similar to our study in which 3 tube with cornual block on HSG proved to have patent tube in SSG \& laparoscopic chromopertubation.

Nabil El-Tabbakh $\mathrm{M}$ and Slamka $\mathrm{P}$ conducted a prospective study: Transvaginal Sonohysterography (TVSH), Versus Hysterosalpingography (HSG) And Laparoscopy in which they evaluated the role of TV-SH in the assessment of tubal patency and compared these results with those obtained using HSG and laparoscopy. They concluded that it could be added to the usual conventional procedures to assess tubal patency as a new, easy and safe method and can be performed as an outpatient procedure in the routine infertility clinic. ${ }^{24}$

Panchal S and Nagori C in their study for assessment of tubal status and said that HSG is painful and inconvenient for patient. Laparoscopy is considered to be the gold standard for tubal evaluation, but is an operative procedure and needs anesthesia. SSG can demonstrate a patent tube but if blocked, the site of block cannot be demonstrated.

A comparative study of the efficacy of sonosalpingography and hysterosalpingogram to test the tubal patency in all women with primary and secondary infertility conducted by Malik B et al showed that for evaluation of tubal patency, SSG had sensitivity of $95.83 \%$ and specificity of $100 \%$ for evaluation of tubal patency. In contrast, in HSG for evaluation of tubal patency, the sensitivity is $91.67 \%$ and specificity is $100 \%$ and concluded that Sonohysterosalpingography appears to be inexpensive, minimally invasive, quick, of no risk of ionizing radiation, and well-tolerable first-line diagnostic method for determining the tubal status and uterine cavity. $^{25}$

In another recent study conducted by Maheux-Lacroixetal S, Sono-HSG and HSG were directly compared with laparoscopy by chromopertubation $\&$ found differences between the two techniques were not statistically significant with a $\mathrm{P}$ value of 0.4 . In our study also, there is significant agreement between the two tests and the diagnostic accuracy of sono-HSG and HSG was comparable with no significant difference in performance of the two tests.

Sambharam $\mathrm{K}$ et al assessed whether sonosalphingography can be used to test tubal patency in infertility patients which is less invasive instead of diagnostic laparoscopy with chromopertubation which is associated with many complications and concluded that sonosalpingography is a simple, convenient and effective method in evaluation of fallopian tube patency. It is much less invasive than diagnostic laparoscopy. However tubal block suspected on sonosalpingography needs confirmation by diagnostic laparoscopy. Sonosalpingography has good value as a screening test for tubal patency on OPD basis.

\section{CONCLUSION}

Ability of SSG to detect tubal patency is at least equal or slightly higher than HSG.SSG can be regarded as a procedure for initial evaluation of uterine cavity and of tubal patency in infertile patients as first line of diagnostic approach as a new, easy and safe method on OPD basis. This saves the patient of radiation exposure as well as pain of contrast administration used in HSG. However tubal block suspected on sonosalpingography needs confirmation by diagnostic laparoscopy.

\section{Funding: No funding sources \\ Conflict of interest: None declared \\ Ethical approval: Not required}

\section{REFERENCES}

1. Zegers-Hochschild F, Nygren KG, Adamson GD, de Mouzon J, Lancaster P, Mansour R et al. The International Committee for Monitoring Assisted Reproductive Technology (ICMART) and the World Health Organization (WHO) revised glossary on ART terminology, 2009. Hum Rep. 2009;24(11):2683-7.

2. World Health Organization. Fertility and infertility. India; WHO;2014. Sexual and reproductive health; Infertility definitions and terminology; 2014. Available from: http://www.who.int/reproductivehealth/topics/infertil ity/definitions/en/.

3. Rastogi R. Role of imaging in female infertility. Indian J Radiol Imaging]. 2010;20(3):168-73. 
4. Lall SS, Ghosh D, Saha D, Bhattacharya AR, Ghosh S, Mitra S. Comparative evaluation of sonosalpingography hysterosalpingography, and laparoscopy for determination of tubal patency. J ObstetGynecol India. 2007;57(2):158-61.

5. Sambharam K, Rode S, Bhalerao A. Comparison of sonosalphingography and laparoscopy in evaluation of tubal patency in infertility patients. International Journal of Scientific and Research Publications. 2015;5(7):1-5.

6. Padubidri VG, Daftary SN. Howkins and Bourne Shaw's textbook of Gynaecology 15th edition. India: Elsevier; 2011. 210p.

7. Khalaf Y. Tubal subfertility. BMJ. 2003;327(7415):610-3.

8. Pandit SP, Adhikari BB, Pandit S. Comparison of sono-salpingography and X-ray hysterosalpingography in the sub-fertile women. PMJN. 2011;11(1):9-11.

9. Simpson WL, Beitia LG, Mester J. Hysterosalpingography: a reemerging study. RadioGraphics. 2006;26(2):419-31.

10. Nor HM, Jayapragasam KJ, Abdullah BJJ. Diagnostic image quality of hysterosalpingography: ionic versus non-ionic water soluble iodinated contrast media. Biomed Imaging Interv J. 2009;5(3):e29.

11. Verhoeve HR. Diagnostic tests for tubal pathology from a clinical and economic perspective. The University of Amsterdam (UvA); 2013. Available from :http://dare.uva.nl/document/477777.

12. Panchal S, Nagori $C$. Imaging techniques for assessment of tubal status. J Hum Reprod Sci. 2014;7(1):2-12.

13. Lacroix SM, Boutin A, Moore L, Burgeron ME, Bujold E, Laberge $\mathrm{P}$, et al. Hysterosalpingosonography for diagnosing tubal occlusion in subfertile women: a systematic review with meta-analysis. Hum Reprod. 2014;29(5):95363.

14. Dessole S, Farina M, Rubattu G, Cosmi E, Ambrosini G, Battista Nardelli G. Side effects and complications of sonohysterosalpingography. Fertil Steril. 2003;80:620.

15. Lim CP, Hazafa Z, Bhattacharya S, Maheshwari A. Should a hysterosalpingogram be a first-line investigation to diagnose female tubal subfertility in the modern subfertility workup? Hum Reprod. 2011;26(5):967-71.
16. Rai PVS, Rao P, Reddy H. Hysterosalpingography (hsg) - the 'last man standing' among the erstwhile plethora of conventional radiological investigations. 'how we do it' and review of literature. International journal of medical and applied sciences. 2014;3(4):223-33.

17. Hajishafiha M, Zobairi T, Zanjani VR, Ghasemi-Rad M, Yekta Z, Mladkova N.Diagnostic value of sonohysterography in the determination of fallopian tube patency as an initial step of routine infertility assessment. J Ultrasound Med. 2009;28(12):1671-7.

18. Oguntoyinbo AE, Amok AOD and KomolafeOF. Sonographic assessment of fallopian tube patency in the investigation of female infertility in Ilorin, Nigeria. Afr J Reprod Health. 2001;5(1):100-5.

19. Tüfekçi EC, Girit S, Bayirli E, Durmuşoğlu F, Yalti S. Evaluation of tubal patency by trans-vaginal sonosalpingography. Fertile Sterile. 1992;57(2);33640.

20. Suttipichate J, Sroywattana C, Dejthevaporn T, Virojchaiwong P, Sripramote M. Transvaginal saline sonosalpingography for the assessment of tubal patency. Thai J ObstetGynaecol. 2002;14(3):223-9.

21. Case AM, Pierson RA. Clinical use of sonohysterography in the evaluation of infertility. J ObstetGynaecol Can. 2003;25(8):641-8.

22. Mona Z, Radu V, Cristian A. Infertility investigation through saline infusion sonohysterosalpingography. Mædica A Journal of Clinical Medicine. 2007;2(1):10-7.

23. Goynumer G, Yetim G, Gokcen O, Karaaslan I, Wetherilt L, Durukan B. Hysterosalpingography, laparoscopy or both in the diagnosis of tubal disease in infertility. World Journal of Laparoscopic Surgery. 2008;1(2):23-6.

24. ObGyn.net. Egypt and Kuwait: El-Tabbakh MN and Slamka P; 2011 Jul 05. Transvaginal sonohysterography (Tv-Sh),Versus hysterosalpingography (Hsg) And laparoscopy. Available from: http://hcp.obgyn.net/ laparoscopy/content/article/1760982/1896801\#

25. Malik B, Patil S, Boricha BG, Kurkal N, Chaoudhry M. A comparative study of the efficacy of sonosalpingography and hysterosalpingogram to test the tubal patency in all women with primary and secondary infertility. Ultrasound Q. 2014;30(2):13943.

Cite this article as: Madhok R, Taneja V. Role of sonosalpingogram in correlation to hysterosalpingogram in assessment of infertility. Int $\mathbf{J}$ Reprod Contracept Obstet Gynecol 2016;5:1936-43. 